CASE REPORT

\title{
Cardiac metastasis from a bronchial carcinoid: report of a case presenting with diffuse thickening of the left ventricular wall
}

\author{
M J Goddard, C Atkinson
}

J Clin Pathol 2004;57:778-779. doi: 10.1136/icp.2004.016055

Bronchial carcinoids are low grade malignancies with an excellent prognosis and a low incidence of metastases. This report describes a case of a typical carcinoid tumour with metastases to the spine after four years and to the heart after eight years. Carcinoid tumours rarely metastasise to the heart and previous reports have identified gastrointestinal primaries as the source in most cases. This is the first published case of typical carcinoid tumour that metastasised to the heart from a bronchial primary.

$\mathrm{T}$ his report describes the case of a typical carcinoid tumour with metastases to the spine after four years and to the heart after eight years. This is the first published case of typical carcinoid tumour that metastasised to the heart from a bronchial primary.

\section{CASE REPORT}

A 56 year old man was referred to Papworth Hospital, Cambridge, UK in early 2003 with a history of progressive exertional dyspnoea and mild orthopnoea. There were no other cardiac symptoms and no history of chest pain. He had previously undergone a left lower lobectomy for a bronchial carcinoid in January 1995 and suffered a metastatic recurrence to his thoracic spine in March 1999. No further recurrence had been seen since that time on follow up.

Examination revealed a soft ejection systolic murmur and there were bibasal crepitations on chest auscultation.

A full blood count, renal function tests, and liver function tests were unremarkable. A chest radiograph revealed cardiomegaly and an electrocardiogram demonstrated some $\mathrm{T}$ wave changes in the precordial leads. A transthoracic echocardiogram revealed a grossly thickened left ventricular wall with poor contractility, which reflected diastolic dysfunction. A provisional clinical diagnosis of cardiac amyloid was made.

The patient was admitted for cardiac catheterisation and endomyocardial biopsy.

Left heart catheterisation showed a cardiac output of $4.1 \mathrm{litre} / \mathrm{min}$ and a cardiac index of $1.9 \mathrm{litre} / \mathrm{min} / \mathrm{m}^{2}$. His left ventricular end diastolic pressure was raised at $40 \mathrm{~mm} \mathrm{Hg}$. Right heart catheterisation showed right ventricular pressures of $70 / 22 \mathrm{~mm} \mathrm{Hg}$, with a mean right atrial pressure of $17 \mathrm{~mm} \mathrm{Hg}$ and a mean pulmonary capillary wedge pressure of $40 \mathrm{~mm} \mathrm{Hg}$. A right ventricular endomyocardial biopsy was performed and showed myocardium and thickened endocardium infiltrated by a tumour composed of monotonous polygonal cells forming small nests and trabeculae (fig 1). No amyloid was demonstrated on staining with Congo red. On immunohistochemical staining, the infiltrating tumour was positive for chromogranin A, PGP9.5, and neural cell adhesion molecule (fig 2). There was no evidence of necrosis and no mitoses were seen.

The histological material from the original lobectomy specimen and the spinal metastasis was reviewed and showed a typical carcinoid tumour, measuring $2.4 \mathrm{~cm}$ in maximum dimension with a similar immunohistological profile. There was lymphatic and perineural invasion within the original lung resection, but no lymph node metastases.

A diagnosis of bronchial carcinoid metastatic to the heart was made.

\section{DISCUSSION}

Bronchial carcinoid tumours tend to behave as low grade malignancies and are usually confined to the lung. Regional lymph node metastases are present in approximately $5 \%$ of cases, resulting in a reduction in the disease free survival at five years from $94-100 \%$ without nodal involvement to $71-$ $74 \%$ with nodal involvement. ${ }^{1}$ Metastatic disease arising from bronchial carcinoids is relatively uncommon. ${ }^{2}$ In this case, lymphatic invasion was present in the original tumour, increasing the risk of dissemination. It is possible, as a result of sampling, that atypical areas with greater nuclear pleomorphism, necrosis, and a higher mitotic rate were missed, but both the vertebral and cardiac metastases showed no atypical features. Furthermore, the prolonged time course of the disease would not be expected in cases of atypical carcinoid or well differentiated neuroendocrine carcinoma.

Metastatic tumours to the heart are relatively uncommon and are usually associated with widespread dissemination and more aggressive tumour types; most often carcinomas of

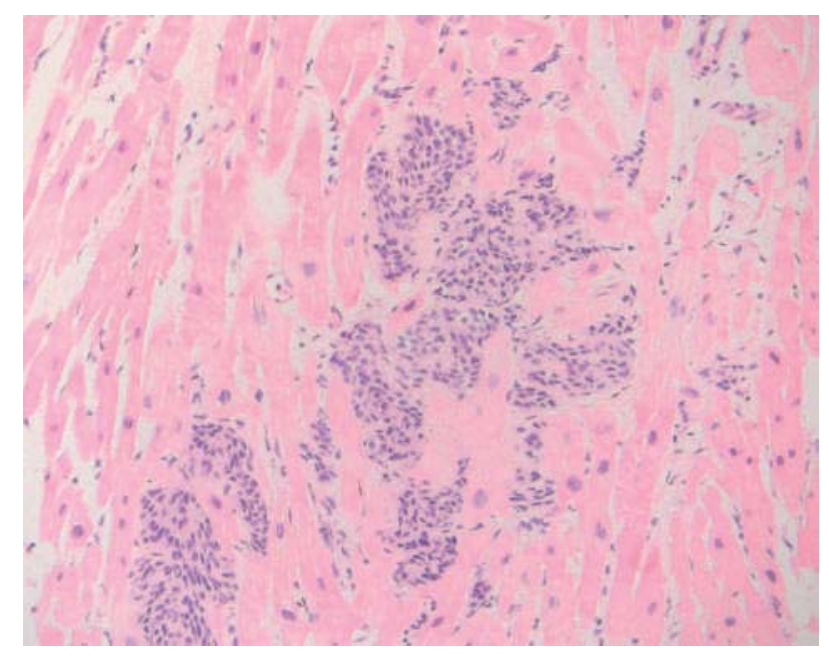

Figure 1 Endomyocardial biopsy showing endocardial and myocardial infiltration by carcinoid (original magnification, $\times 100$ ). 


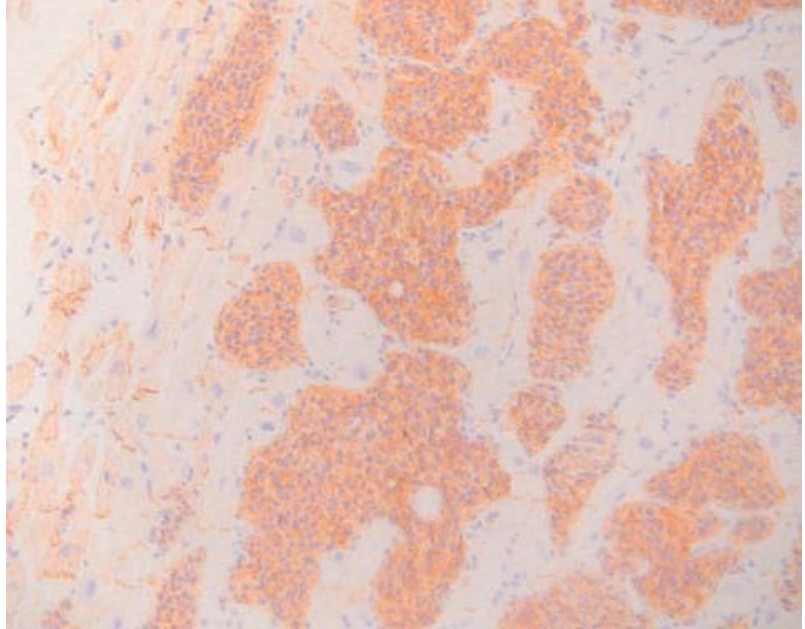

Figure 2 Immunohistochemical staining for neural cell adhesion molecule (original magnification, $\times 400$ ).

the lung and breast, and melanomas. There are several case reports and small series of metastatic carcinoid involving the heart. Most have arisen from primary sites in the gastrointestinal tract, predominantly the ileum. Two cases were reported in 1997 of atypical bronchial carcinoids with intracardiac metastases. ${ }^{3}$ In a study of 74 patients with carcinoid heart disease, three patients with metastases to the myocardium were described on echocardiographic appearance. ${ }^{4}$ The cases reported have usually demonstrated a discrete mass on echocardiography, rather than the diffuse wall thickening seen in this case. ${ }^{4-7}$ Nearly all cases were associated with the carcinoid syndrome and had hepatic metastases, although one case without hepatic disease has been reported. ${ }^{5}$ Carcinoid heart disease with left sided valvular lesions associated with bronchial carcinoids is rare, with an incidence of between $1 \%$ and $7 \%{ }^{8}$ In this case, there was no evidence of valvular lesions on echocardiography. Metastatic disease from bronchial carcinoids is uncommon, but in a large epidemiological study was seen in approximately 7\% of cases, although there was no separation of typical and atypical carcinoid tumours.'

"The longterm outlook for this patient is unclear, but survival with myocardial metastases from ileal carcinoids is usually short, although occasional patients have survived for up to 12 years"
Take home messages

- We report the first case of a typical bronchial carcinoid metastasising to the myocardium

- Carcinoid tumours rarely metastasise to the heart and previous reports have identified gastrointestinal primaries as the source in most cases

This case report is the first report of a typical bronchial carcinoid metastasising to the myocardium. The indolent nature of the disease is highlighted by the prolonged time course. Unlike gastrointestinal carcinoids, which produce discrete masses on echocardiography, this case showed only diffuse thickening of the ventricular wall. The longterm outlook for this patient is unclear, but survival with myocardial metastases from ileal carcinoids is usually short, although occasional patients have survived for up to 12 years. ${ }^{6}$

\section{Authors' affiliations}

M J Goddard, C Atkinson, Department of Pathology, Papworth Hospital, Cambridge CB3 8RE, UK

Correspondence to: M J Goddard, Department of Pathology, Papworth Hospital, Cambridge CB3 8RE, UK; martin.goddard@papworth.nhs.uk

Accepted for publication 7 January 2004

\section{REFERENCES}

1 Francioni F, Rendini EA, Venuta F, et al. Low grade neuroendocrine tumours of the lung (bronchial carcinoids) -25 years experience. Eur J Cardiothorac Surg 1990;4:472-6.

2 Modlin I, Sandor A. An analysis of 8305 cases of carcinoid tumours. Cancer 1997;79:813-29.

3 Drake WM, Jenkins PJ, Phillips RR, et al. Intracardiac metastases from neuroendocrine tumours. Clin Endocrinol 1997;46:517-22.

4 Pellikka PA, Tajik J, Khandheria BK, et al. Carcinoid heart disease. Clinical and echocardiographic spectrum in 74 patients. Circulation 1993;87:1188-96.

5 Kasi VS, Ahsanudin AN, Gilbert C, et al. Isolated metastatic myocardial carcinoid tumour in a 48 year old man. Mayo Clin Proc 2002;77:591-4

6 Pandya UH, Pellikka PA, Enriquez-Sarano M, et al. Metastatic carcinoid tumour to the heart: echocardiographic-pathologic study of 11 patients. J Am Coll Cardiol 2002;40:1328-32.

7 Sciller VI, Fishbein MC, Siegel RJ. Unusual cardiac involvement in carcinoid syndrome. Am Heart J 1986; 1 12:1322-3.

8 Hasleton PS. Spencer's pathology of the lung, 5th ed. McGraw-Hill, 1996.

9 SEER cancer statistics review. Bethesda (MD): US Department of Health and Human Sciences, Public Health Institute, National Institute of Health, National Cancer Institute, 1993. 